\title{
Impact of Shear Strain and Quantum Confinement on $<110>$ Channel nMOSFET with High-Stress CESL
}

H. Takashino, T. Okagaki, T. Uchida, T. Hayashi, M. Tanizawa, E. Tsukuda, K. Eikyu, S. Wakahara, K. Ishikawa, O. Tsuchiya, Y. Inoue

Renesas Technology Corp., 4-1 Mizuhara, Itami, Hyogo 664-0005, Japan

\{takashino.hiroyuki|okagaki.takeshi\}@ renesas.com

\begin{abstract}
Numerical study in conjunction with comprehensive bending experiments has demonstrated that (100)-Si has the optimum channel direction along $\langle 110\rangle$ in terms of the device performance of strained 65nm-node nMOSFETs with Contact Etch Stop Layer (CESL), and that both the shear strain component and the quantum confinement effect play an important role in this superiority.
\end{abstract}

\section{Introduction}

The process-induced strain has become one of the principal technology boosters for improving MOSFET mobility and has led to great concerns about the selection of the optimal channel direction [6,7]. In state-of-the-art devices, the stress distribution is complicated as shown in Fig.1 [1]. Therefore, it is necessary to optimize the device structure with the support of physical analysis using TCAD. In this study, we investigate the dependence of the performance gain of nMOSFETs on channel direction at various stress levels ranging from 4-point bending experiments ( $100 \mathrm{MPa})$ to high-stress CESL ( $500 \mathrm{MPa})$. A newly developed mobility model, which takes into account the shear strain component and the quantum confinement effect has, for the first time, provided a consistent explanation of stress effects on device characteristics.

\section{Model}

To obtain the new model, we extended a simple mobility model for strain effect in $\mathrm{Si}$ band structure [2], to include both the shear strain and the quantum confinement effect in the inversion layer of nMOSFETs. Let's begin by looking at the Bir-Pikus effective Hamiltonian near the $\mathrm{X}$ point [3]. Considering the Si crystal symmetry, the Hamiltonian of the $\mathrm{z}$-valley is given by

$$
\frac{\hbar^{2}}{2 m_{t}}\left(k_{x}^{2}+k_{y}^{2}\right)+\frac{\hbar^{2}}{m_{t}} s k_{x} k_{y} \varepsilon_{x y}+\frac{\hbar^{2}}{2 m_{l}} k_{z}^{2}+\triangle E_{z}(\varepsilon)
$$

where $m_{t}\left(0.19 m_{0}\right)$ and $m_{l}\left(0.98 m_{0}\right)$ represent transverse and longitudinal electron mass, respectively. $\triangle E_{z}(\varepsilon)$ is the conduction band deformation potential with the parameters $D, U$. The second term is the origin of difference between longitudinal piezo-coefficient $\pi_{l}$ and transverse piezo-coefficient $\pi_{t}$ in the $<110>$ channel. The parameter $s$, which is omitted in [2], expresses the dependence of effective mass on shear strain $\varepsilon_{x y}$. The lowest subband energy $E_{z}$ is expressed as $C_{q m}\left(m_{0} / m_{l}\right)^{1 / 3}(q F)^{2 / 3}$ by the conventional quantization method for the inversion layer. The $\mathrm{z}$-axis is taken as the confinement direction, $F$ is the confinement electric field and $C_{q m}$ is a constant parameter. Similarly, the lowest subband energy of x-valley $E_{x}$ and y-valley $E_{y}$ is given by $C_{q m}\left(m_{0} / m_{t}\right)^{1 / 3}(q F)^{2 / 3}$. Considering the quantum confinement effect according to [4], the mobility tensor with constant relaxation time is given by

$$
\mu_{x x}=\frac{p_{z}}{m_{t}}+\frac{p_{x}}{m_{l}}+\left(1-s^{2} \varepsilon_{z x}^{2}\right) \frac{p_{y}}{m_{t}}, \mu_{x y}=s \varepsilon_{x y} \frac{p_{z}}{m_{t}}, \mu_{y y}=\frac{p_{z}}{m_{t}}+\left(1-s^{2} \varepsilon_{y z}^{2}\right) \frac{p_{x}}{m_{t}}+\frac{p_{y}}{m_{l}} .
$$


The $p_{i}$ in the above equations is the electron occupancy of the valley $i$ which is expressed as $p_{i}=I_{i} / \sum_{i=x_{2}, z} I_{i}$ with

$$
I_{x}=\frac{\exp \left\{-\left(E_{x}+\triangle E_{x}(\varepsilon)\right) / k \mathrm{~T}\right\}}{\sqrt{1-s^{2} \varepsilon_{y z}^{2}}}, I_{y}=\frac{\exp \left\{-\left(E_{y}+\triangle E_{y}(\varepsilon)\right) / k \mathrm{~T}\right\}}{\sqrt{1-s^{2} \varepsilon_{z x}^{2}}}, I_{z}=\frac{\exp \left\{-\left(E_{z}+\triangle E_{z}(\varepsilon)\right) / k \mathrm{~T}\right\}}{\sqrt{1-s^{2} \varepsilon_{x y}^{2}}} .
$$

The expression (2) is suitable for a Drift-Diffusion (D.D.) simulator. In implementation of our model, we used the scalar type mobility $\mu$ given by $\mu=\sqrt{\sum_{i}\left(\sum_{j} \mu_{i j} \partial_{j} E_{f}\right)^{2}} / \sqrt{\sum_{i}\left(\partial_{i} E_{f}\right)^{2}}$ with the gradient of the quasi-Fermi level $E_{f}$, because of the conformity with the conventional discretization scheme of the D.D. simulator.

It should be noted that, since our model has been derived from the effective Hamiltonian (1), we can easily obtain the mobility expression of the arbitrary orientation by the coordinate transformation of the Hamiltonian.

\section{Results \& Discussion}

Figs. 2, 3 show the 4-point-bending method measurement results for the $<100>$ and $<110>$ channels, respectively. $\delta I d / I d$ changes linearly in the small strain region at about $0.1 \%$ and the piezo-coefficient can be well-defined from the slope. The coefficient of $<100>$ gets smaller as the gate bias becomes larger, while the gate bias dependence of the $<110>$ channel is negligible.

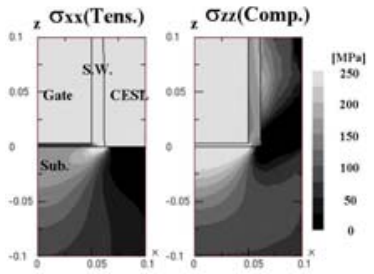

Figure 1: A stress distribution of nMOSFET with CESL. $\sigma_{x x}$ (left figure) and $\sigma_{z z}$ (right figure) have nonuniform distribution.

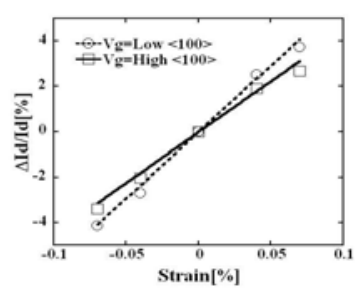

Figure 2: $<100>$ strain dependence of nMOSFET current measured at two different gate biases.

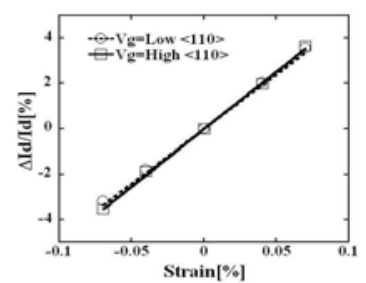

Figure 3: $<110>$ strain dependence of nMOSFET current measured at two different gate biases.

Fig. 4 shows the gate bias dependence of the piezo-coefficients obtained by 4-point bending method and that calculated by D.D. simulator using our model. The simulation results well reproduce all the measured data, with a clear separation between $\pi_{l}$ and $\pi_{t}$ in the $\langle 110\rangle$ channel which can't be expressed by the model in [2]. The parameters used here are summarized in Table 1. The calculated piezo-coefficients at the weak confinement limit are close to the bulk values of [5] (Table 2). In order to select the

\begin{tabular}{|c|c|c|c|}
\hline$D$ & $U$ & $s$ & $C_{q m}$ \\
\hline-8.6 & 10.0 & 45.0 & $9.0 \mathrm{e}-6$ \\
\hline
\end{tabular}

Table 1: Parameter values. Unit of $D$ and $U$ is [eV], $s$ is dimensionless. Unit of $C_{q m}$ is $\left[\mathrm{eV}^{1 / 3} \mathrm{~cm}^{2 / 3}\right]$.

\begin{tabular}{|r|c|c|c|}
\hline & \multicolumn{2}{|c|}{ This model } & {$[5]$} \\
\hline & $\mathrm{Vg}=5 \mathrm{~V}$ & bulk limit & bulk \\
\hline$<100>\pi_{l}$ & -36 & -93 & -102 \\
$\pi_{t}$ & 7.5 & 46 & 53 \\
\hline$<110>\pi_{l}$ & -26 & -30 & -31 \\
$\pi_{t}$ & -2 & -18 & -17 \\
\hline
\end{tabular}

Table 2: Piezo-coefficients from this model and bulk values in [5]. Unit is $\left[10^{-12} \mathrm{~cm}^{2} / \mathrm{dyn}\right]$. 
optimum channel direction for the current gain [6], it should be noted that, under high gate bias, $\pi_{l}$ in $\langle 110\rangle$ is larger than that in $\langle 100\rangle$ with high tensile strain while $\pi_{l}$ in $<110\rangle$ is less than that in $<100\rangle$ with low tensile strain applied by using the 4-point bending method (Fig. 5, [7]).

Fig. 6 shows the current enhancement of the nMOSFET with tensile CESL. The calculation results for this structure obtained by [8] were also plotted and are almost in complete agreement with the measurements. We find that, in the high tensile region with CESL, the current enhancement of the $<110>$ channel is greater than that of $<100>$. The difference between the $\langle 110\rangle$ mobility and that of $\langle 100\rangle$ is mainly derived from the $\mu_{x y}$ of (2) which becomes larger with the greater shear tensile strain $\varepsilon_{x y}$ applied with the $\langle 110\rangle$ tensile stress of CESL. In Fig. 7, it can be seen that the conventional

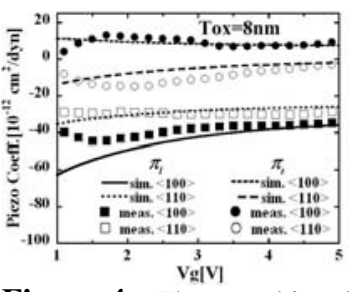

Figure 4: The gate bias dependence of $\pi_{l}$ and $\pi_{t}$ for Figure 5: The calculated Tox $=8 \mathrm{~nm}$ nMOSFET. The re- gate bias dependence of $\pi_{l}$ for sults calculated with our model $\langle 100\rangle$ and $\langle 110\rangle$. The deare also plotted and well-agree pendence under high tensile with the measured data. The stress (b) differs from that unparameter values are summa- der low tensile stress (a). rized in Table 1.
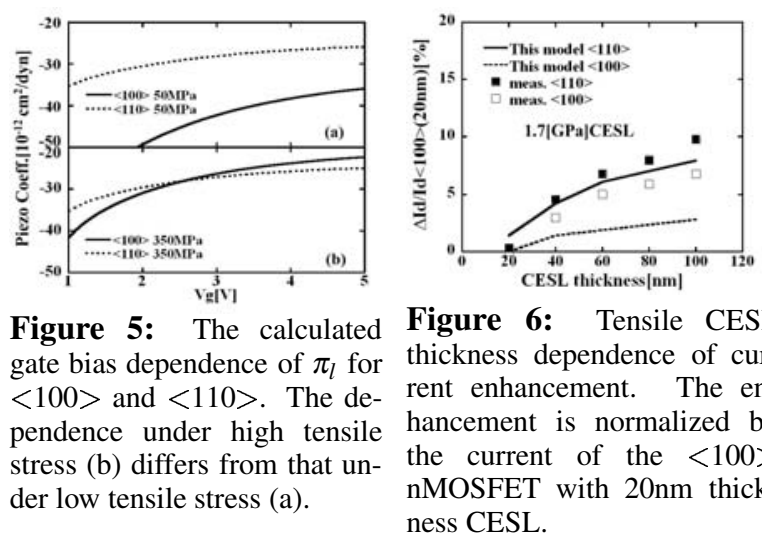

Figure 6: Tensile CESL hickness dependence of current enhancement. The enhancement is normalized by the current of the $\langle 100\rangle$ nMOSFET with $20 \mathrm{~nm}$ thickness CESL.

model [2] gives channel direction dependence opposite to the measurement results and our calculated results. It is clear that considering both shear strain and quantum confinement effects is essential for estimating nMOSFET performance with CESL. Fig. 8 shows nonuniform mobility enhancement and confinement electric field $F$ along the $<110>$ channel. Because of the large $F$, enhancement in the source-side channel region becomes smaller than that in the other regions.

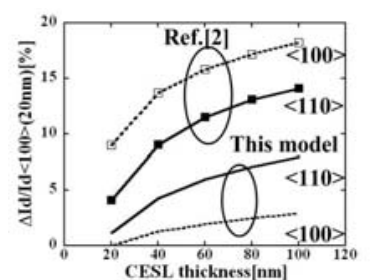

Figure 7: The effect of shear strain and quantum confinement on the same nMOSFET structure used in Fig.6. We compare our extended model with the model in [2].

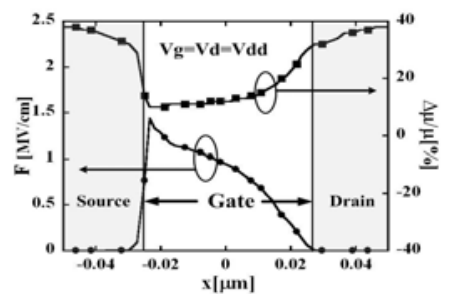

Figure 8: The confinement electric field $F$ and mobility enhancement distribution along $<110>$ of $100 \mathrm{~nm}$ thickness CESL. In the strong confinement region $(x \sim-0.025 \mathrm{um})$ the enhancement is small while that of the S/D region is large.

The quantum confinement greatly changes the temperature dependence of mobility and 
current enhancement (Figs. 9, 11). Under tensile stress with CESL, the enhancement in the $\langle 110\rangle$ channel depends less on temperature than in $\langle 100\rangle$ (Fig. 9). Fig. 11 shows the temperature dependence of the nMOSFET current reduction with compressive stress induced by STI (Fig. 10). In the $<100>$ channel case, the results obtained with our model well-agree with the measurement data. Similarly, under tensile stress, the temperature dependence of current variation in $\langle 110\rangle$ is more stable than in $<100\rangle$.

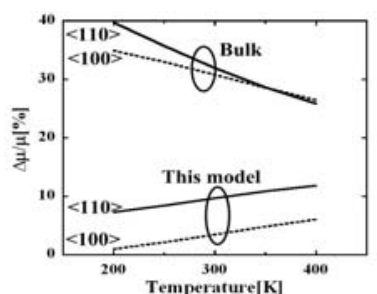

Figure 9: Temperature de- Figure 10: A stress distripendence of mobility enhance- bution of the nMOSFET with ment at the channel center of STI. STI gives compressive $100 \mathrm{~nm}$ thickness CESL. The stress at about 600MPa along dependence of the inversion the channel while at about layer is opposite to that of bulk. 100MPa along the vertical.

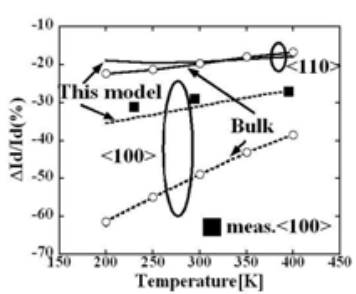

Figure 11: Temperature dependence of nMOSFET current reduction with STI stress. The dependence of the inversion layer is less than that of bulk.

\section{Conclusion}

We have proposed a novel mobility model derived directly from the Bir-Pikus effective Hamiltonian. Using this model, we have shown the advantage of $<110>$ channel nMOSFETs with high-stress CESL, while accurately reproducing the gate bias dependence of higher $\langle 100\rangle$ piezo-coefficients in the weak-stress of the bending experiment elucidated in [5]. The robustness of $<110>$ channel nMOSFETs against temperature fluctuation has also been revealed by using this model.

\section{Acknowledgements}

The authors would like to thank Dr. N. Mori and Dr. Y. Kamakura for the valuable discussions regarding the Bir-Pikus effective Hamiltonian.

\section{References}

[1] T. Uchida et al.,Proc.SISPAD 2005, p.199, 2005.

[2] J. L. Egley and D. Chidambarrao, Solid-St. Elec., p.1653, 1993.

[3] G. L. Bir and G. E. Pikus, "Symmetry and Strain-Induced Effects in Semiconductors," ed. Wiley, New York, 1974.

[4] M. J. van Dort, P. H. Whoerlee and A. J. Walker, Solid-St. Elec., vol.37, p.411, 1994.

[5] S. M. Sze, "Semiconductor Sensors," ed. Wiley, New York, 1994.

[6] H. Irie, K. Kita, K. Kyuno and A. Toriumi, IEDM Tech. Dig., p.255, 2004.

[7] K. Uchida, T. Krishnamohan, K. C. Saraswat and Y. Nishi, IEDM Tech. Dig., p.135, 2005; M. Hane, T. Ikezawa, M. Kawada, T. Ezaki and T. Yamamoto, Proc.SISPAD 2006, p.55, 2006.

[8] SELETE 3D process simulator HySyProS. 